\title{
Do mating strategies determine genital allometry in African mole rats (Bathyergidae)?
}

\author{
A. A. Kinahan ${ }^{1,2}$, N. C. Bennett ${ }^{1}$, L. E. Belton ${ }^{1} \&$ P.W. Bateman ${ }^{1}$
}

1 Department of Zoology and Entomology, Mammal Research Institute, University of Pretoria, Pretoria, South Africa

2 Department of Zoology, School of Animal, Plant and Environmental Sciences, University of Witwatersrand, Johannesburg, Wits, South Africa

Correspondence

Philip W. Bateman, Department of Zoology and Entomology, Mammal Research Institute, University of Pretoria, Pretoria 0002, South Africa.

Email: pwbateman@zoology.up.ac.za

\section{Abstract}

Allometry describes the relationship of components of an organism with change in overall body size and has become the focus of numerous studies on the evolution of genitalia. Typically, negative allometry is observed in insects and is explained by stabilizing selection whereas the very few studies on mammals have shown a positive allometric relationship of genitalia in the body size, thought to have arisen as a result of sexual selection. However, all mammal species studied to date are thought to use mainly post-copulatory mating strategies. Across mammals, however, both pre-and post-copulatory strategies occur (although the two are not mutually exclusive). We propose that where pre-copulatory strategies are mainly used, no reproductive benefits would result from evolving positively allometric genitalia. As such, mammal genitalia are not typically positively allometric but rather allometry will, to a certain degree, be determined by mating strategy. We tested this prediction using four species of African mole rats (Bathyergidae) exhibiting variation in their life histories and mating strategies. Although generally supported, in that positive allometry did not occur in species that we assumed use mainly pre-mating strategies, positive allometry did not occur in either of the promiscuous species thought to use post-copulatory strategies. We suggest, therefore, that while mating strategies may tentatively determine genital allometry, whether positively allometric genitalia occur also depends on a number of complex interacting factors. In addition, this study provides further evidence and empirical support for the co-evolution of male and female genitalia in mammals.

\section{Introduction}

Allometry describes change in relative dimensions of components of an organism correlated with changes in size (Gayon, 2000). Over the past decade, allometric studies investigating the relative size of genitalia in relation to body size have become the focus of considerable research with regard to genital evolution. Whereas, the majority of studies have primarily focused on insects (e.g. Arnqvist, 1998; Eberhard et al., 1998; Hosken, Minder \& Ward, 2005), more recent studies have begun to 
examine the allometry of genitalia in mammals (Miller \& Burton, 2001; Lüpold, McElligott \& Hosken, 2004; Kinahan et al., 2007), although the baculum has been the focus of many studies (e.g. Patterson \& Thaeler, 1982; Patterson, 1983; Lessa \& Cook, 1989; Lariviére \& Ferguson, 2002). In insects, a negative allometric relationship of genitalia with change in size appears to be the rule rather than the exception. This pattern is thought to have arisen as a result of stabilizing selection (Eberhard et al., 1998). The 'one-size-fits-all' hypothesis (Eberhard et al., 1998) proposes that selecting for intermediate-sized genitalia irrespective of body size allows males to copulate with average-sized females.

In contrast, the few studies conducted on mammals (Miller \& Burton, 2001; Lüpold et al., 2004) and the only study examining both male and female genitalia (Kinahan et al., 2007), all have shown a positive allometric intra-specific relationship between genitalia and body size, which may suggest that mammalian genitalia are typically positively allometric. Positive allometry results when the net benefit of investing in a structure is greater than the net benefit of being larger (Green, 1992; Petrie, 1992) and several studies have shown that positive allometry occurs for sexually selected traits (e.g. Alatalo, Höglund \& Lundberg, 1988; Petrie, 1988; Simmons \& Tomkins, 1996). Positive allometry of mammalian genitalia therefore should incur reproductive advantages particularly in mating systems where mainly post-copulatory strategies are used, for example in multi-mating systems (polygyny and polyandry) or when copulation is through coercion, thereby increasing the male's competitive ability either for mate acquisition or paternal success. By post-copulatory, we primarily mean how females react to a mating event that has either been forced on them (Clutton-Brock \& Parker, 1995), over which they have had little chance to evaluate the male with which they mate, except retrospectively through subsequent matings (Bateman, Gilson \& Ferguson, 2001), or through promoting sperm competition. For example, females may cryptically select which males to favour by estimating the size of their genitalia (Thornhill, 1983; Eberhard, 1996) if, that is, genitalia are an indicator of quality. Positive allometry in female genitalia could also be beneficial by potentially enabling the female greater control over fertilization under sperm competition (Birkhead, 1995), thus creating a more difficult journey for sperm and ensuring that only the fittest are likely to reach their ova (Anderson, Dixon \& Dixon, 2006; Kinahan et al., 2007). In response to this, males may have evolved proportionally longer penises to facilitate this indirect sperm selection by ensuring that sperm is deposited as close as possible to the site of fertilization. Such co-evolution of genitalia is likely to arise from sexual conflict (Arnqvist, 1997), as evolutionary reproductive interests differ between males and females (Hosken \& Stockley, 2005).

There are several mammalian examples where post-copulatory strategies are not used but instead mating success may be determined by mainly pre-copulatory strategies (although the two strategies are not mutually exclusive: for example male 'coercion' could be seen as a pre-copulatory mechanism if the coercive methods themselves are used as the method of cryptic choice by the female) (see Andersson, 1994). Under primarily pre-copulatory strategies, mating pairs may be decided by direct male-male competition, with the winners obtaining mating access to females or females may select mates based on some ornamental criteria as an indicator of good genes (Andersson, 1994). In such instances, it is unlikely that the cost of investing in proportionally larger genitalia would incur any further reproductive benefits. As a result, we anticipate that in mammals where pre-copulatory strategies are utilized, 
positive allometric genitalia should not be evident but rather, along with other morphological traits, genitalia should show an isometric relationship with body size (increasing proportionally with an increase in body size) or have no relationship. Because all studies on genital allometry in mammals to date have used species where mainly post-copulatory strategies are assumed to occur (Miller \& Burton, 2001; Lüpold et al., 2004; Kinahan et al., 2007), we therefore suggest that positive allometry is not the 'rule' for mammalian genitalia but rather that genital allometry in mammals will be determined by their mating strategies.

African mole rats (Bathyergidae) provide an excellent model to test the hypothesis that mating strategy determines genital allometry in mammals because a variety of life history and mating strategies occur within this family. We used four species (and two sub-species) that ranged from strictly solitary through to eusocial, seasonal to aseasonal breeders, induced and spontaneous ovulators and those where a variety of mating strategies are evident. Using both male and females of each species, we examined genital allometry in the Cape dune mole rat Bathyergus suillus, the Cape mole rat Georychus capensis, the Damaraland mole rat Cryptomys damarensis and the Natal and highveld mole rats Cryptomys hottentotus natalensis and Cryptomys hottentotus pretoriae. The Natal and highveld mole rats are phylogenetically similar as are their life histories and strategies. As a result, data for these two sub-species were pooled and are hereafter referred to as Cryptomys hottentotus. We predicted that in the solitary species (B. suillus and G. capensis) where polygyny and polyandry occur and matings are largely through coercion (males seeking out females and forcing them to copulate) (Bennett \& Jarvis, 1988; Jarvis \& Bennett, 1991; Clutton-Brock \& Parker, 1995), post-copulatory strategies will come into play and there will be reproductive advantages in investing in proportionally larger genitalia. We therefore predicted the occurrence of positive allometric genitalia in both these species. In contrast, within the social species (C. hottentotus and C. damarensis) where typically (but not always) monogamous pair bonds are formed (Burland et al., 2002, 2004; Bishop et al., 2004), usually determined by body size (pre-copulatory), we predicted that there would be no reproductive advantages in investing in proportionally longer genitalia; hence, we anticipate that positively allometric genitalia will not be evident but rather an isometric or no relationship will occur between genitalia and body size. Furthermore, if male and female genitalia co-evolve (Arnqvist, 1997), we expect males and females of the same species to exhibit the same allometric patterns in their genitalia.

\section{Materials and methods}

\section{Data collection}

The Cape dune mole rats B. suillus were captured at Cape Town International Airport, Cape Town, South Africa (33 $\left.58^{\prime} \mathrm{S} 18^{\circ} 37^{\prime} \mathrm{E}\right)(\mathrm{n}=87)$. The Cape mole rats G. capensis were captured at Darling, Cape Town $\left(33^{\circ} 22^{\prime} \mathrm{S} 15^{\circ} 25^{\prime} \mathrm{E}\right)(\mathrm{n}=21)$. The Natal mole rats C. h. natalensis were captured in Glengarry, Kwazulu, Gauteng Natal $\left(25^{\circ} 58^{\prime} \mathrm{S}\right.$ $21^{\circ} 49^{\prime} \mathrm{E}$ ), and the highveld mole rats C. h. pretoriae were captured in Tygerpoort $\left(25^{\circ} 48^{\prime} \mathrm{S} 28^{\circ} 21^{\prime} \mathrm{E}\right)$ (both sub-species together, $\mathrm{n}=40$ ). The Damaraland mole rats $\mathrm{C}$. damarensis were captured in Hotazel, Northern Cape Province $\left(27^{\circ} 17^{\prime} \mathrm{S} 22^{\circ} 58^{\prime} \mathrm{E}\right)$ $(n=56)$. All the mole rats were captured under permit from the respective Nature 
Conservation authorities and were captured using Hickman live-traps (Hickman, 1979). All specimens used in this study were collected during their breeding seasons. All individuals used in this study were adults; however, in C. damarensis the sexes were separated into breeders (those forming pair bonds) and non-breeders (those remaining in colony). We were unable to distinguish clearly between breeding and non-breeding animals for $\mathrm{C}$. hottentotus and so it is likely that the dataset included both. All individuals of G. capensis and B. suillus were considered breeders. Table 1 shows a summary of the life histories for each of the species examined in this study.

Table 1 Summary of life history traits for each species used in the study (after Bennett et al., 1991; Bennett \& Faulkes, 2000)

\begin{tabular}{llllr}
\hline & Bathyergus suillus & Georychus capensis & Cryptomys damarensis & Cryptomys hottentotus \\
\hline Sociality & Solitary & Solitary & Social & Social \\
Sexual dimorphism & Yes & No & Yes & No \\
Breeding & Seasonal & Seasonal & Aseasonal & Seasonal \\
No. of litters per year & 2 & 2 & 4 & 2 \\
Mean litter size & $2(1-4)$ & $6(4-10)$ & $3(1-5)$ & $2(1-3)$ \\
Birth mass (g) & 34 & $5-12$ & $8-10$ & $8-11$ \\
Mean adult body mass (g) & 780 & 180 & 110 & 90 \\
Ovulation & Induced & Induced & Spontaneous & Induced \\
Mean colony size & Non-colonial & Non-colonial & $12(2-41)$ & $6(2-16)$ \\
\hline
\end{tabular}

Summary data for B. suillus were taken from Kinahan et al. (2007). All genital measurements were taken using digital callipers to two decimal places (mm). We examined repeatability by measuring a representative sample from each trait twice and conducted regression analysis of measurement one on measurement two (Hosken et al., 2005). We found that all measurements were highly repeatable, with each exhibiting $\mathrm{r}^{2}$ values $>0.98$. Body length was used as an indicator of body size and was measured from the tip of the nose to the base of the tail (animal placed ventrally on a flat surface, tail slightly raised). Genital measurements included the length of the penis and the female reproductive tract. Once specimens were defrosted, the penis was dissected out of the males and measured ex situ. Female reproductive tract length was measured by cutting open the abdomen of each specimen and inserting a probe into the vagina and measuring the distance from the point of insertion to the point where the uterine horns separated.

\section{Data analysis}

Our data were log-transformed to meet the assumptions of normality, after which we carried out ordinary least square (OLS) analyses to examine relationships between traits where slopes of the regression line were tested for a significant deviation from a slope of zero $\left(\beta_{0}\right)$. If our OLS regression indicated a relationship between genitalia and body size, we then performed a reduced major axis (RMA) regression to test for the nature of their allometric relationships (Lüpold et al., 2004; Kinahan et al., 2007). An advantage of using RMA over OLS to determine allometric relationships is that RMA is appropriate to overcome scale dependence and when both independent and dependent variables are subject to measurement error (Sokal \& Rohlf, 1995).

Furthermore, because there is some discrepancy in the literature as to which method is best to examine such data (Eberhard, Huber \& Rodriguez, 1999), we present both methods. For RMA regressions, we used t-tests to examine deviations of the slope of 
the regression line from a slope of one, $\left(\beta_{1}\right)$. We inferred isometry when $\beta=1$ : positive allometry when $\beta>1$ and negative allometry when $\beta<1$.

\section{Results}

Our data agreed with those documented previously in the literature (Bennett \& Faulkes, 2000) in that sexual dimorphism occurred in B. suillus $(\mathrm{t}=7.45$, d.f. $=107$, $\mathrm{P}<0.001)$ and between breeding $\mathrm{C}$. damarensis individuals only $(\mathrm{t}=4.18$, d.f. $=25$, $\mathrm{P}<0.001$ ) and that $\mathrm{B}$. suillus was significantly larger than the other three species (Kruskal-Wallis, $\mathrm{H}=98.6, \mathrm{n}=4, \mathrm{P}<0.001$, post hoc $\mathrm{P}<0.001$ ).

Summary statistics for our OLS and RMA analyses are shown in Table 2. We found no OLS relationship between genitalia and body size in either males or females in C. hottentotus or breeding C. damarensis individuals. However, we observed a strong relationship between genitalia and body size in the non-breeding males and females in C. damarensis and subsequently found this to be isometric. Although we also observed significant relationships between body size and genitalia in both sexes of the solitary species B. suillus and G. capensis, the nature of their allometric relationship differed. Positive allometry was found in B. suillus whereas an isometric relationship occurred in G. capensis. We found that there were no differences in the allometric slopes between male $\mathrm{G}$. capensis and $\mathrm{C}$. damarensis non-breeders $(\mathrm{t}=0.88$, d.f. $=23$, $\mathrm{P}=0.39)$ or between the females of the species $(\mathrm{t}=1.7$, d.f. $=23, \mathrm{P}=0.09)$.

Table 2 Summary statistics for ordinary least squares (OLS, testing for a significant deviation from a slope of zero, $\beta_{0}$ ) and reduced major axis regression (RMA, testing for a significant deviation from a slope of one, $\beta_{1}$ ) analyses for four African mole-rat species

\begin{tabular}{|c|c|c|c|c|c|c|c|c|c|}
\hline Species & Sex & Reproduction status & $n$ & $r$ & $\beta_{0} \pm S E$ & t-value & $\beta_{1} \pm \mathrm{SE}$ & t-value & Allometry \\
\hline \multirow[t]{4}{*}{ Cryptomys damarensis } & Female & Breeders & 12 & 0.34 & $1.47 \pm 1.29$ & $1.14 \mathrm{NS}$ & & & \\
\hline & Male & Breeders & 14 & -0.49 & $-1.26 \pm 0.65$ & $-1.94 \mathrm{NS}$ & & & \\
\hline & Female & Non-breeders & 14 & 0.63 & $1.1 \pm 0.4$ & $2.79^{*}$ & $1.76 \pm 0.4$ & $1.92 \mathrm{NS}$ & Isometry \\
\hline & Male & Non-breeders & 16 & 0.67 & $1.12 \pm 0.33$ & $3.37^{* *}$ & $1.67 \pm 0.33$ & $2.03 \mathrm{NS}$ & Isometry \\
\hline \multirow[t]{2}{*}{ Cryptomys hottentotus } & Female & Unknown & 30 & 0.18 & $0.15 \pm 0.06$ & $0.46 \mathrm{NS}$ & & & \\
\hline & Male & Unknown & 10 & 0.11 & $0.15 \pm 0.47$ & 0.33 NS & & & \\
\hline \multirow[t]{2}{*}{ Georychus capensis } & Female & Breeders & 11 & 0.84 & $1.2 \pm 0.26$ & $4.65^{* *}$ & $1.43 \pm 0.26$ & $1.66 \mathrm{NS}$ & Is ometry \\
\hline & Male & Breeders & 10 & 0.80 & $0.91 \pm 0.24$ & $3.77^{* *}$ & $1.14 \pm 0.24$ & $0.53 \mathrm{NS}$ & Isometry \\
\hline \multirow[t]{2}{*}{ Bathyergus suillus } & Female & Breeders & 29 & 0.53 & $1.71 \pm 0.53$ & $3.21^{* *}$ & $3.26 \pm 0.53$ & $4.23^{* * *}$ & Positive \\
\hline & Male & Breeders & 58 & 0.583 & $1.6 \pm 0.3$ & $5.37^{* *}$ & $2.74 \pm 0.3$ & $5.85^{* * *}$ & Positive \\
\hline
\end{tabular}

${ }^{a}$ Data taken from (Kinahan et al., 2007).

The sample size (n) and the correlation coefficients (r) are also shown here. RMA was carried out only if OLS regression showed a significant relationship between traits. Isometry was inferred when $\beta_{1}=1$; positive, when $\beta_{1}>1$ and negative allometry if $\beta_{1}<1$. Genitalia (y-axis) were regressed against body length ( $\mathrm{x}$-axis).

OLS, ordinary least square; RMA, reduced major axis; NS, non-significant; se, standard error.

${ }^{*} \mathrm{P}<0.05 ;{ }^{* *} \mathrm{P}<0.01 ;{ }^{* * *} \mathrm{P}<0.001$. 


\section{Discussion}

Based on the limited studies carried out to date on genital allometry in mammals, it may be assumed that, just as negative allometric genitalia appears to be the rule rather than the exception in insects, mammals, conversely, may typically exhibit positive allometric genitalia. The present study has revealed that in African mole rats, this does not appear to be the case but rather that positive allometry may be an exception to the rule. As predicted, our study showed that in primarily monogamous species where pre-copulatory strategies are likely to be used, no evidence of positive allometric genitalia was observed. However, our data did not support our prediction that positive allometric genitalia will occur in promiscuous systems where primarily post-copulatory mating strategies occur. It is likely therefore that its prevalence will be influenced by a number of complex interacting factors in addition to a species mating strategy. This study further highlights evidence for the co-evolution of genitalia in mammals.

Several reproductive advantages may be incurred by having positive allometric genitalia, all of which are associated with maximizing fitness in particular where variation in paternity may occur (Kinahan et al., 2007). Furthermore, because many other sexually selected traits have shown positive allometry, sexual selection rather than the pleiotropy hypothesis (Mayr, 1963) may more appropriately explain the finding of positive allometry in mammalian genitalia. There are three main ways in which positive allometric genitalia may incur reproductive advantages (1) A longer penis may be beneficial if females cryptically select mates and use penis size as an indicator of male quality (cryptic female choice) (Thornhill, 1983; Eberhard, 1996). (2) Longer penises may be able to deposit sperm closer to the uterine horns and so are more likely to fertilize the ova (sperm competition) (Birkhead, 1995). (3) Females may elongate their reproductive tracts, making it more difficult for sperm, thereby ensuring only the fittest are likely to fertilize their ova. In response to this, males may evolve proportionally longer penises (sexual conflict) (Arnqvist, 1997). None of these post-copulatory strategies are necessarily mutually exclusive.

We predicted therefore that across the four species, positive allometry should be evident in the solitary species only where multi-mating and post-copulatory strategies are likely to occur, but not in the social species where pair bonds are formed via precopulatory mate choice and where they are typically, but not exclusively, monogamous. Although we found that positively allometric genitalia were not evident in either of the social species, C. damarensis and C. hottentotus, and positive allometry did occur in the solitary B. suillus (Kinahan et al., 2007), it was not, as predicted, evident in the other solitary species, G. capensis, which exhibited isometric genitalia (both the penis and the vagina increase in direct proportion to body size). One possible explanation for this is that G. capensis typically has much larger litter sizes (4-10) than the other species in this study and particularly compared with the other solitary species, B. suillus (1-4) (Bennett et al., 1991). Georychus capensis is also frequently observed on the surface where they incur greater mortality. As a consequence, females may not invest in elongating their reproductive tract because they invest their resources in producing larger litters. In response to this, males demonstrate isometric genitalia (or potentially no relationship) as they are not under selective pressure to have positively allometric penises. It thus follows that, in order 
to compete for paternity, males may use other mechanisms, for example, they may have developed disproportionately larger testicular size for sperm competition (Harcourt et al., 1981; Gage \& Freckleton, 2002). However, further studies are needed to validate this assumption. In addition, it should also be noted that another lifehistory trait differs between the two solitary species: B. suillus, but not G. capensis being sexually dimorphic, suggesting a much lower intensity of sexual selection in G. capensis.

As predicted, however, when pre-copulatory mating strategies are used we did not observe positive allometric genitalia. The breeders within C. damarensis colonies exhibited no allometric relationship. Colonies of C. damarensis usually, but not exclusively, constitute a monogamous breeding pair, with the reproductive animals being the largest of each sex, and a number of related but socially suppressed individuals of either sex, although small males traditionally classed as 'non-breeders' can also sire young (Burland et al., 2002). It is usually the older and larger animals that disperse (Hazell et al., 2000; Scantlebury et al., 2006) and therefore pair bonds are determined by body size. Because it is usually the largest individuals that are breeders, we would expect to find no relationship between body size and genitalia, probably due to the biased representation of disproportionately larger-bodied animals occurring within the breeders. As expected, non-breeding animals of both sexes within these colonies exhibited isometry, whereby genitalia increased in direct proportion to an increase in body size. As Burland et al. (2002) found that males other than the large 'breeders' can also sire young in the colony, it is possible that the breeding females may either promote sperm competition through extra-pair copulations, or, less likely, that males can coerce females.

Although, as predicted, C. hottentotus did not exhibit positive allometry, we observed no relationship between genitalia and body size. We were unable to distinguish between breeders and non-breeders in this dataset and thus our results may be confounded by this. Kinahan et al. (2007) observed a positive allometric relationship between vagina depth and body size in B. suillus only during the breeding season. Male genitalia measures were, however, not influenced by season. However, we would expect that should reproductive status differentiation be possible for our C. hottentotus specimens, our results would be likely to mirror those found in the $\mathrm{C}$. damarensis. The observed results could be complicated by the plasticity of the sociality of C. hottentotus. Both the sub-species combined in this study are found in areas that tend to mesic conditions, where it might be expected that sociality will break down, giving more opportunities for multiple-mating, sperm competition and cryptic female choice (Bishop et al., 2004).

All the species in this study are induced ovulators, except $\mathrm{C}$. damarensis, which is a spontaneous ovulator (Table 1). Bathyergus suillus and G. capensis males have spinose penises, C. h. natalensis has small protrusions on the penis and C. damarensis has ridges but no other ornamentation on the penis (Parag et al., 2006). The spines and protrusions may have a role in inducing ovulation. In addition, the large, sharp spines of B. suillus, combined with the positively allometric penis, may have a role in causing mild physiological trauma in the females, discouraging them from mating again, thereby reducing sperm competition risk. 
This study suggests that, at least in African mole-rats, genitalia, like many other morphological traits, are generally isometric and deviations from isometry may result from variations in mating strategies. This study also provides further evidence and strong empirical support for the co-evolution of male and female genitalia in mammals. We suggest that, in mammals, positive allometric genitalia are not the general rule but rather the exception. We further suggest that positively allometric genitalia are less likely to occur in species that use mainly pre-copulatory choice strategies. However, while we infer that positive allometry is likely to occur in multimating systems where both polygyny and polyandry are evident (due to postcopulatory strategies being used), we acknowledge that it is more complex and other factors such as litter sizes, and/or sexual dimorphism for example, may actual determine its prevalence. Pre-and post-copulatory mate choice strategies are unlikely to occur in isolation from each other. It is also likely that in polyandrous species, sexual selection is more intense than in monandrous species, with the result that, along with other adaptations, positive allometry of the sexually selected traits is more likely to be observed (Eberhard, 1985). Further data from diverse taxa would be valuable.

\section{References}

- Alatalo, R.V., Höglund, J. \& Lundberg, A. (1988). Patterns of variation in tail ornament size in birds. Biol. J. Linn. Soc. 34, 363-374.

- Andersson, M. (1994). Sexual selection. Princeton, NJ: Princeton University Press.

- Anderson, M.J., Dixon, A.S. \& Dixon, A.F. (2006). Mammalian sperm and oviducts are sexually selected: evidence for co-evolution. J. Zool. (Lond.) 270, 682-686.

- Arnqvist, G. (1997). The evolution of animal genitalia: distinguishing between hypotheses by single species studies. Biol. J. Linn. Soc. 60, 365-379.

- Arnqvist, G. (1998). Comparative evidence for the evolution of genitalia by sexual selection. Nature 393, 784-786.

- Bateman, P.W., Gilson, L.N. \& Ferguson, J.W.H. (2001). Male size and sequential mate preference in the cricket Gryllus bimaculatus. Anim. Behav. 61, 631-637.

- Bennett, N.C. \& Faulkes, C.G. (2000). African mole-rats ecology and eusociality. Cambridge. UK: Cambridge University Press.

- Bennett, N.C. \& Jarvis, J.U.M. (1988). The reproductive biology of the Cape mole-rat, Georychus capensis (Rodentia, Bathyergidae). J. Zool. (Lond.) 214, 95-106.

- Bennett, N.C., Jarvis, J.U.M., Aguilar, G.H. \& McDaid, E.J. (1991). Growth rates and development in six species of African mole-rats (Family: Bathyergidae). J. Zool. (Lond.) 225, 13-26.

- Birkhead, T.R. (1995). Sperm competition: evolutionary causes and consequences. Repro. Fertil. Dev. 7, 755-775.

- Bishop, J.M., O'Ryan, C., Spinks, A.C., Bennett, N.C. \& Jarvis, J.U.M. (2004). Molecular insight into patterns of colony composition and paternity in the common mole-rat, Cryptomys hottentotus hottentotus: a subterranean social mammal. Mol. Ecol. 13, 1217-1229. 
- Burland, T.M., Bennett, N.C., Jarvis, J.U.M. \& Faulkes, C.G. (2002).

Eusociality in African mole-rats: new insights from patterns of genetic relatedness in the Damaraland mole-rat. Proc. Roy. Soc. Lond. Ser. B 269, 1025-1030.

- Burland, T.M., Bennett, N.C., Jarvis, J.U.M. \& Faulkes, C.G. (2004). Colony structure and parentage in wild colonies of cooperatively breeding Damaraland mole-rats suggest incest avoidance alone may not maintain reproductive skew. Mol. Ecol. 13, 2371-2379.

- Clutton-Brock, T.H. \& Parker, G.A. (1995). Sexual coercion, harassment and intimidation in animal societies. Anim. Behav. 49, 1345-1365.

- Eberhard, W.G. (1985). Sexual selection and animal Genitalia. Cambridge Massachusetts, and London: Harvard University Press.

- Eberhard, W.G. (1996). Female control: sexual selection by female cryptic choice. Princeton: Princeton University Press.

- Eberhard, W.G., Huber, B.A. \& Rodriguez, R.L. (1999). Don't forget the biology: a reply to Green. Evolution 53, 1624-1627.

- Eberhard, W.G., Huber, B.A., Rodriguez, R.L., Briceno, R.D., Salas, I. \& Rodrigeuz, V. (1998). One size fits all? Relationships between the size and degree of variation in genitalia and other body parts in twenty species of insects and spiders. Evolution 52, 415-431.

- Gage, M.J.G. \& Freckleton, R.P. (2002). Relative testis size and sperm morphometry across mammals: no evidence for an association between sperm competition and sperm length. Proc. Roy. Soc. Lond. Ser. B 270, 625-632.

- Gayon, J. (2000). History of the concept of allometry. Am. Zool. 40, 748-758.

- Green, A.J. (1992). Positive allometry is likely with mate choice, competitive display and other functions. Anim. Behav. 43, 170-172.

- Harcourt, A.H., Harvey, P.H., Larson, G.S.G. \& Short, R.V. (1981). Testis weight, body weight and breeding system in primates. Nature 293, 55-57.

- Hazell, R.W.A, Bennett, N.C., Jarvis, J.U.M. \& Griffin, M. (2000). Adult dispersal in the cooperatively breeding Damaraland mole-rat, Cryptomys damarensis: a case study from the Waterberg region of Namibia. J. Zool. (Lond.) 252, 19-26.

- Hickman, G.C. (1979). A livetrap and trapping technique for fossorial mammals. S. Afr. J. Zool 14, 9-12.

- Hosken, D.J., Minder, A.M. \& Ward, P.I. (2005). Male genital allometry in Scathophagidae (Diptera). Evol. Ecol. 19, 501-515.

- Hosken, D.J. \& Stockley, P. (2005). Sexual conflict. Curr. Biol. 15, R535R536.

- Jarvis, J.U.M. \& Bennett, N.C. (1991). Ecology and behavior of the family Bathyergidae. In The biology of the naked mole-rat: 66-96. Sherman, P.W., Jarvis, J.U.M. \& Alexander, R.D. (Eds). Princeton, NJ: Princeton University Press.

- Kinahan, A.A., Bennett, N.C., O'Riain, M.J., Hart, L. \& Bateman, P.W. (2007). Size matters: genital allometry in an African mole-rat (Family: Bathyergidae). Evol. Ecol. 21, 201-213.

- Lariviére, S. \& Ferguson, S.H. (2002). On the evolution of the mammalian baculum: vaginal friction, prolonged intromission or induced ovulation? Mammal Rev. 32, 283-294.

- Lessa, E.P. \& Cook, J.A. (1989). Interspecific variation in penial characters in the genus Ctenomys (Rodentia: Octodontidae). J. Mammal. 70, 856-860. 
- Lüpold, S., McElligott, A.G. \& Hosken, D.J. (2004). Bat genitalia: allometry, variation and good genes. Biol. J. Linn. Soc. 83, 497-507.

- Mayr, E. (1963). Animal species and evolution. Cambridge: Harvard University Press.

- Miller, E.H. \& Burton, L.E. (2001). Its all relative: allometry and variation in the baculum (os penis) of the harp seal, Pagophilus groenlandicus (Carnivora: Phocidae). Biol. J. Linn. Soc. 72, 345-355.

- Parag, A., Bennett, N.C., Faulkes, C.G. \& Bateman, P.W. (2006). Penile morphology of African mole rats (Bathyergidae): structural modification in relation to mode of ovulation and degree of sociality. J. Zool. (Lond.) 270, 323-329.

- Patterson, B.D. (1983). Baculum-body size relationships as evidence for a selective continuum on bacular morphology. J. Mammal. 64, 496-499.

- Patterson, B.D. \& Thaeler, C.S. Jr. (1982). The mammalian baculum: hypotheses on the nature of bacular variability. J. Mammal. 63, 1-15.

- Petrie, M. (1988). Intraspecific variation in structures that display competitive ability: large animals invest relatively more. Anim. Behav. 36, 1174-1179.

- Petrie, M. (1992). Are all secondary sexual display structures positively allometric and, if so why? Anim. Behav. 43, 173-175.

- Scantlebury, M., Speakman, J.R., Oosthuizen, M.K., Roper, T.J. \& Bennett, N.C. (2006). Energetics reveals physiologically distinct castes in a eusocial mammal. Nature 440, 795-797.

- Simmons, L.W. \& Tomkins, J.L. (1996). Sexual selection and the allometry of earwig forceps. Evol. Ecol. 10, 97-104.

- Sokal, R.R. \& Rohlf, F.J. (1995). Biometry. 3rd edn. San Francisco: WH Freeman.

- Thornhill, R. (1983). Cryptic female choice and its implications in the scorpionfly Harpobittacus nigriceps. Am. Nat. 122, 765-788. 